\title{
ISO 50001 Sistema de Gestión Energética 2018 Guía de Implementación
}

\author{
ISO 50001 The New Version 2018 Implementation Guide
}

\author{
Á. M. Buritica-Macías iD ; J G. López-Quintero iD ; C. A. Buriticá-Noreña iD \\ DOI: https://doi.org/10.22517/23447214.22761 \\ Artículo de reflexión
}

\begin{abstract}
Resumen - Esta guía permite la iniciación del proceso de mejoramiento de desempeño energético en las empresas de acuerdo a la norma de sistema de gestión de la energía ISO 5001:2018, norma de gran impacto en la actualidad en todos los tipos de empresas. Se realizó una revisión a la norma incluyendo las herramientas necesarias para el mejoramiento de la eficacia energética y el uso del consumo racional de la energía, incluyendo los aspectos principales a trabajar a nivel organizacional para alcanzar estos objetivos. Su aplicación permitirá la reducción de los gases de efecto invernadero, los impactos ambientales relacionados y la reducción de los costos de la energía a través de una gestión sistemática de la energía; con el potencial para ser un medio importante en la reducción del calentamiento global y mejorar el compromiso de las empresas respecto al medio ambiente.
\end{abstract}

Palabras claves - consumo energético, dirección estratégica, línea de base energética, mejora continua, objetivos energéticos.

Abstract-This guide allows the initiation of the energy performance improvement process in companies according to the energy management system standard ISO 5001: 2018, a standard that currently has great impact in all types of companies. A revision of the standard was carried out, including the tools necessary to improve energy efficiency and the use of rational energy consumption, including the main aspects to work at the organizational level to achieve these objectives. Its application will allow the reduction of greenhouse effect gases, the related environmental impacts and the reduction of energy costs through a systematic energy management; with the potential to be an important means in reducing global warming and improving the commitment of companies to the environment.

Index Terms - continuous improvement, energetic base line, energy consumption, energy management system, energy planning, energetic risk, strategic direction.

\section{INTRODUCCIÓN}

$\mathrm{L}$ A adopción de un sistema de gestión energética como la ISO 50001:2018 es una decisión voluntaria estratégica, que es obligatoria en varios países del mundo, su implementación

Este manuscrito fue enviado el 29 de julio de 2020 y aceptado 23 de junio 2021.

Á. M. Buritica-Macias, Ingeniería Industrial Universidad Tecnológica de Pereira, Colombia, (amburitica@utp.edu.co). no depende del tipo, tamaño o clase de una organización, es una herramienta pensada para reducir el calentamiento global. Entre más sea el compromiso de eliminar contaminantes energéticos, más se reducen los costos de energía y el impacto ambiental, de esta manera las organizaciones pueden cumplir con los objetivos de desarrollo de sostenibilidad específicos legales con el medio ambiente, un compromiso mundial que nos compete a todos. La segunda versión de esta norma se publicó en agosto del año 2018, y debido a que es nueva no existe una guía para su implementación. Este documento servirá para que las organizaciones implementen la norma.

\section{METODOLOGÍA}

\section{A. Clausula 1. Objeto y campo de aplicación}

Todos los requisitos son aplicables para establecer, implementar, y mejorar un sistema de gestión de la energía en todo tipo de organizaciones, permitiendo alcanzar una mejora continua en su desempeño energético. Esto incluye el uso, consumo y eficiencia de la energía, la medición, documentación, procesos, y las personas involucradas que contribuyen al desempeño de este sistema de gestión [1].

\section{B. Clausula 2 Referencias normativas}

De acuerdo con la norma no se tienen referencias normativas, pero se debe cumplir con la estructura de alto nivel de la norma ISO 9001:2015 de sistemas de gestión [2].

\section{Clausula 3 Términos y definiciones}

La norma tiene 28 definiciones, que además hacen parte de la norma de calidad ISO 9001, y añade significados como el de desempeño energético. Estas definiciones son una guía de términos energéticos y se deben consultar cuando sea requeridas [3].

C. A. Buriticá-Noreña., Ingeniería Industrial Universidad Tecnológica de Pereira, Pereira Colombia (caburitica@utp.edu.co).

G. López-Quintero, Ingeniería Industrial Universidad Tecnológica de Pereira, Pereira Colombia (jlq2012@utp.edu.co) 


\section{Clausula 4 Conocimiento de la organización y su contexto}

Las organizaciones deben identificar las situaciones internas y externas que tienen impacto potencial negativo para alcanzar los resultados planificados en su sistema de gestión de la energía, esto se realiza por medio de la identificación de las debilidades y amenazas, considerando los entornos legales, económicos, culturales, sociales, tecnológicos, de mercado, de productividad, etc. No se debe dejar por fuera los aspectos internos o externos a Colombia, y todas aquellas situaciones específicas que no permiten lograr las metas energéticas planificadas, todo lo que ocasione no cumplimiento se debe programar para la mejora de la administración del riesgo [4].

Es importante entender lo que desean y lo que esperan los empleados, accionistas, proveedores, clientes, y todos los que de una u otra forma están relacionados con la institución de la empresa. Se debe identificar y conocer de qué manera puede afectar el sistema de administración energética.

Para describir el alcance del sistema de administración de la energía, la organización específica sus productos y servicios y los requisitos de las partes interesadas. Para facilitar su manejo se sigue considerando la estructura por procesos.

\section{E. Clausula 5 Liderazgo}

La alta dirección debe demostrar el liderazgo y el compromiso con el sistema de gestión de la energía, lo cual se evidencia con la participación, seguimiento a la política de calidad de la energía y a los objetivos y metas energéticas, asegurando que estén de acuerdo con la planificación estratégica y el contexto de la organización, estos deben ser divulgados. Se debe promover la toma de conciencia de los empleados con el sistema de gestión de la energía, asignando los recursos financieros y de personal, con el objetivo de la mejora continua. La alta dirección debe asignar los recursos económicos que requiere la administración del sistema, haciendo seguimiento al programa, además debe firmar la política de energía haciendo seguimiento a las metas planteadas.

La política energética debe estar disponible para los clientes y proveedores, los colaboradores de la organización y las partes interesadas la deben conocer; ella debe ir en concordancia con lo que es el negocio y sirve de marco para la revisión de los objetivos y metas energéticas de la organización [5].

La alta dirección debe nombrar el representante de la dirección por el sistema de gestión de la energía, quien es el responsable de mantener informada a la gerencia del desempeño del sistema. Las funciones, responsabilidades y la autoridad de los cargos que afectan el sistema deben ser conocidas por los empleados, se requiere que el personal las entienda para cumplir con las obligaciones asignadas.

\section{F. Clausula 6 Planificación}

Se debe hacer un programa para identificar los posibles riesgos que puedan interferir para no cumplir con los programas de la administración energética, estos deben ser valorados con una propuesta de mejora continua para evitar que lleguen a ocurrir. En dicho programa se debe analizar los aspectos que afectan a la organización en la gestión energética. Ya sea de orden interno o externo, como se identifican en el numeral 4 de la norma.

La meta es disminuir y evitar las situaciones que originen el incumplimiento de las metas energéticas; se debe elaborar unos principios como la política empresarial del riesgo en donde deben estar incluidos los objetivos energéticos propuestos, y a estos se les debe incluir en el programa de administración del riesgo, localizando en que parte del proceso se pueden presentar. Al mostrar el riesgo se reconoce si es de origen económico, o por causas ambientales, financieras, o administrativas, etc. Igualmente se tienen en cuenta los aspectos internos o externos, local, regional, nacional, e internacional. Posteriormente se debe hacer un estudio de las causas y efectos que pueden incidir en el programa de la administración de la energía y el cumplimiento de los objetivos energéticos.

En el estudio se debe asignar la posibilidad que ocurra algún riesgo, se debe identificar el control o mecanismos que tiene la empresa para que no se vea afectada por uno de estas circunstancias imprevistas. De acuerdo con esos estudios, se hace la valoración del riesgo, aplicando fórmulas y gráficas que determinan el orden de atención de dicha situaciónn. Se deben tomar las acciones de mejora continua del sistema de gestión de la energía, aprovechando las oportunidades, describiendo la estrategia a implementar, el responsable de la implementación, el tiempo y la meta con los indicadores que se pretenden. De esta manera se elimina o reduce el riesgo residual que queda después de su administración para la mejora continua del sistema de gestión de la energía.

Se debe establecer una línea base de indicadores del consumo energético que sirva como referencia para fijar los objetivos energéticos, se deben establecer para cada sección de la empresa, clasificados por proceso y estos deben ser divulgados a todos los niveles de la organización [6]. Deben ser coherentes con la planificación estratégica y con los principios de la política energética, medibles, alcanzables con las metas, asignando responsables de administrarlos, con fechas de cumplimiento y se les debe hacer seguimiento, actualizándolos cuando se requieran y lo más importante, definir el camino para alcanzarlos.

Los resultados alcanzados al finalizar los periodos de metas, son el insumo importante para la evaluación de desempeño del sistema de gestión de la energía y la planificación para nuevos periodos y si se requiera cambiar, se deben asignar los recursos necesarios de orden económico o humano con nuevas responsabilidades necesarias para cumplir los objetivos energéticos planteados.

\section{G. Clausula 7 Apoyo}

La institución debe asignar el presupuesto financiero y el personal necesarios para implementar y sostener el sistema de administración de la energía. La institución debe asignar los recursos para capacitar y actualizar los funcionarios que realicen tareas para ella o en su nombre relacionadas con usos 
significativos de la energía, que sean competentes, con base en una educación, formación, habilidades y experiencia adecuadas.

Se debe hacer un plan con las necesidades de capacitación requerida, ya sea por actualización de tecnología o aplicando mejora continua de los empleados, con el resultado de sus evaluaciones anuales. La organización debe establecer manuales de funciones y responsabilidades con los requisitos por educación, experiencia y competencias, además de técnicas relacionadas con el uso y manejo responsable de la energía, asegurando así que la organización contrate sus empleados con los requisitos establecidos [7].

La toma de conciencia con una encuesta similar a la de clima organizacional de debe incluir preguntas sobre el conocimiento del programa de administración energética, como por ejemplo: si la implementación del sistema de gestión de la energía ha contribuido a mejorar su desempeño, conocimiento de los objetivos energéticos, los indicadores con metas del sistema de gestión energético, si se entiende la política de la energía y como es su contribución al sistema de administración de la energía. La encuesta es una anual como mínimo, y sirve para medir el compromiso de los empleados con el sistema y aplicar la mejora continua cuando se requiera.

En cuanto a la comunicación tanto interna como externa de todo lo que afecta el sistema, debe definirse quienes son los autorizados para hacer las comunicaciones, el contenido de lo que se va a comunicar, y la organización decide que se debe comunicar con respecto al desempeño de los procesos de la gestión de la energía.

La institución debe tener establecido los requisitos para los productos y servicios en el sistema de gestión de la energía que presta, estableciendo procesos de comunicación con los clientes, para entregar sus productos y servicios energéticos como lo solicitaron. Los canales de comunicación donde se reciben las sugerencias deben acoplarse a los requisitos de los productos, servicios y políticas de la empresa, los clientes deben conocerlos, estos pueden estar en los catálogos o en los contratos que firman con ellos. Es necesario especificar cómo tramitar reclamos y quejas para estar en constante mejoramiento.

Deben quedar registrados los requisitos y la información documentada del sistema de gestión de la energía. Es necesario que haya registro sobre los actores autorizados para elaborar, cambiar, eliminar, actualizar, o modificar aquellos documentos, y en la aprobación debe asegurarse que tenga la fecha de vigencia. Se debe especificar si los documentos están en medio magnético o escrito, controlando los registros del sistema de administración de la energía, determinando donde están o quien los administra. Y si se pierden cómo se puede obtener una nueva copia, también como se controla la distribución de los documentos de manejo especial o confidencial, los meses o años que deben estar en circulación y el de retención especificando si son vigentes o no. Igualmente especificar si se deben guardar por ley o por conveniencia de la empresa y cuál es el uso que finalmente se hará de ellos. En los documentos entregados a la empresa por externos de manejo especial que afecten el sistema de administración, se especifica cómo se hace llegar a los diferentes destinatarios. Estos deben tener formatos establecidos, uno para los documentos y otro para los registros, se puede implementar el requisito de la información documentada que afecta el sistema.

\section{H. Clausula 8 Operación}

En el desarrollo y control de la realización del producto, tener en cuenta los riesgos que puedan afectar el cumplimiento del sistema de gestión de la energía, estableciendo los criterios para aceptar los productos y servicios energéticos especificando y planificando los cambios que se hacen a lo programado. En los procedimientos o instructivos del sistema de gestión energético elaborados se debe especificar los puntos de mediciones de energía que se deben realizar durante todos los procesos desde su inicio hasta su final.

La organización debe considerar que el diseño o desarrollo de productos, equipos, procesos, y servicios nuevos no impacten negativamente el programa de gestión energética. Todas las actividades que se realizan deben estar planificadas, incluyendo los elementos de entrada; como encuestas o análisis de mercado que garanticen la producción o desarrollos nuevos de servicios o bienes materiales, o modificaciones en las instalaciones. Tener en cuenta los requisitos legales, reglamentarios y normativos que se deben cumplir, y su impacto ambiental. Se debe estudiar las consecuencias por desviaciones a lo planeado que puedan afectar el desempeño energético. La planificación debe incluir además el control que se debe hacer desde que se inician las actividades hasta que se terminan, haciendo los controles respectivos en todos los procesos durante la producción o en la prestación del servicio, que requieran de un cauteloso seguimiento para evitar afectaciones a largo plazo. La norma exige que se debe hacer la verificación y validación, especificando lecturas de consumos en todos los puntos claves para valorar el desempeño del sistema de gestión de la energía, verificando si se cumple con lo que se planifico en el diseño y desarrollo, o en la solicitud del proyecto.

En la adquisición de servicios de energía, productos, y equipos que tienen un impacto significativo con el uso de la energía, la organización debe considerar y asegurar que se cumpla con los requisitos del sistema de administración de la energía. Deben informar a los proveedores que las compras serán partes evaluadas sobre la base del desempeño energético [8], haciendo una selección de ellos mediante la evaluación de sus productos o servicios en conformidad con el sistema de gestión de la energía, a estos se les debe hacer cada año la evaluación de su cumplimiento, o si continúan siendo apropiados. Se debe dejar la documentación como prueba, teniendo en cuenta que se les debió dar toda la información necesaria para la prestación del servicio, detallando especificaciones técnicas de los productos, en cuanto a los requisitos de calidad energética.

Si se presentan cambios en la producción y la prestación del servicio energético, diferente a lo que inicialmente se planifico, la institución encargada debe documentar dichos cambios, haciendo la actualización de la administración energética para 
asegurar que se sigue cumpliendo lo establecidos en el sistema de administración de la energía.

\section{Clausula 9 Evaluación de desempeño}

La institución define lo necesario para medir y hacer seguimiento que le sirva para evaluar el desempeño energético y lo debe hacer a intervalos planificados, es decir, se debe especificar los usos significativos de la energía y el resultado de la revisión energética, detallando la forma de estudio, y análisis de los productos o servicios de energía, con sus respectivos indicadores. La eficacia para alcanzar los objetivos y las metas energéticas los establece la empresa, midiendo principalmente el consumo real de la energía frente al esperado. Todo lo anterior debe quedar registrado.

Los equipos de medida que están instalados en las máquinas o los que se utilizan para medir el desempeño energético, deben ser los adecuados en su escala y calidad del instrumento y deben estar calibrados por laboratorios competentes para garantizar los resultados. La organización debe definir las necesidades de medición y la frecuencia con la que se deben hacer las revisiones para asegurar que se encuentran funcionando correctamente. De no ser así, se deben definir fechas para enviarlas a calibrar a laboratorios acreditados con la norma ISO- IEC 17025. La institución puede contar con patrones de referencia propios cumpliendo la normatividad, para verificar sus equipos, si se presentan desviaciones en las mediciones de los equipos utilizados que afectan el desempeño energético se debe investigar, hacer seguimiento y deben revisar resultados que se afectaron debido a estas desviaciones.

Los requisitos legales relacionados con su uso y consumo de la energía, deben estar muy claros, completos, y estar bien definidos. Los documentos pactados con partes interesadas, deben cumplir con los requisitos de ley y reglamentarios [9], estos deben estar actualizados. Se debe asegurar que están de acuerdo con las especificaciones de los productos o los servicios energéticos entregados, se debe hacer revisiones con fechas programadas, con las respectivas evidencias escritas.

Para asegurar el cumplimiento de la norma a intervalos planificados se deben programar auditorías internas, se pueden guiar por la norma ISO 19011, esto se debe realizar mínimo una vez por año, es importante hacerlas con auditores internos capacitados de la organización. Se recomienda igualmente revisar todos los requisitos de la norma ISO 50001, se deberían realizar en periodos planificados durante una vigencia anual, asegurando la objetividad e imparcialidad de la auditoria. Es importante auditar siempre el cumplimiento de las metas energéticas planificadas. La dirección debe conocer el estado de las auditorías, debe tomar decisiones para hacer las correcciones y las acciones correctivas necesarias de las desviaciones halladas.

El sistema de la administración de la energía debe ser revisada por la dirección para asegurar su eficacia, incluyendo las revisiones anteriores de auditorías internas y externas, el cumplimiento de proveedores y contratistas, la revisión de la política energética, del personal involucrados con el desempeño de la energía, el estado de los hallazgos encontrados, las acciones correctivas, el manejo de la administración de los riesgos, el estado de los requisitos legales y reglamentarios, además del cumplimiento de los objetivos y metas energéticas del desempeño energético programado para años posteriores. Importante también evaluar el cumplimiento de los requisitos solicitados por los clientes, de los productos y servicios energéticos prestados, las quejas, reclamos recibidos, y todo lo que afecte el desempeño del sistema de administración de la energía.

Los resultados de la revisión por la dirección deben incluir todas las decisiones y acciones tomadas con los cambios en el desempeño energético, cambios en la política energética, cambios en los indicadores de los objetivos y metas energéticas, cambios en los recursos asignados o demás elementos del sistema de la administración de la energía.

\section{J. Clausula 10 Mejora}

El objetivo es la mejora continua del desempeño energético de la institución, sus procesos, productos y servicios de energía prestados y contratados, y las posibles situaciones de afectación. Los hallazgos encontrados se deben estudiar y analizar y son objeto para programar la mejora continua del sistema.

La organización debe identificar los productos y los servicios de energía no conformes de acuerdo a lo planificado, debe identificarlos con letreros para mostrar donde no se cumple y que el personal conozca los aspectos de no conformidad. Se deben realizar las correcciones respectivas y dependiendo de su impacto se realizaran las acciones correctivas.

Para hacer la mejora continua hay que medir y comparar con la línea base de energía, emplear herramientas estadísticas que permitan asegurar la efectividad de las medidas para tomar acciones de oportunidades de mejora y alcanzar los objetivos propuestos dentro del sistema de gestión energética.

\section{CONCLUSIONES}

La implementación de la norma ISO 50001:2018 permite que las organizaciones sustituyan sus procesos, equipos, y materias primas que producen gases de efecto invernadero como el monóxido o bióxido de carbono, por otras amigables con el medio ambiente y así contribuyen a la descarbonización del planeta, y a la reducción significativa del calentamiento global.

La implementación de la norma de gestión energética permite la reducción de los costos energéticos de estas, y por ende importantes ahorros en los costos de producción, que es uno de los componentes más altos en los procesos productivos, haciendo más competitivas a las organizaciones. Implementarla permite a las organizaciones generar una buena imagen corporativa por el compromiso con el medio ambiente, y esto se vuelve una estrategia de mercadeo eficaz para mejorar sus ingresos. 


\section{RECOMENDACIONES}

Por políticas internacionales sobre el calentamiento global que es una preocupación universal, se espera que la implementación de la norma de gestión energética ISO 5001:2018 será de ámbito obligatorio, por este motivo con este artículo las organizaciones pueden iniciar su implementación y no esperar hasta que eso ocurra.

La documentación que tienen las instituciones con otro tipo de normas se debe utilizar para la implementación de la ISO 50001:2018, actualizándola a sus requisitos.

\section{REFERENCIAS}

[1] Instituto Colombiano de Normas Técnicas ICONTEC ,Norma Técnica Colombiana ISO 50001:20011, Sistema de gestión de la energía requisitos orientados para su uso, objeto y campo de aplicación, p.1, 2011

[2] Instituto Colombiano de Normas Técnicas ICONTEC, Norma Técnica colombiana ISO 50001: 2011, Sistema de Gestión de la energía requisitos orientados para su uso, objeto y campo de aplicación, p. 1, 2011

[3] Instituto Colombiano de Normas Técnicas ICONTEC, Norma Técnica Colombiana ISO 50001: 2011, Sistema de Gestión de la energía, términos y definiciones, pp. 2-4, 2011

[4] Instituto Colombiano de Normas Técnicas ICONTEC, Norma técnica Colombia ISO 9001:2015 contexto de la organización, p. 22, 2015

[5] Instituto Colombiano de Normas Técnicas ICONTEC, Norma Técnica colombiana ISO 50011: 2011, Liderazgo, política energética, p. 6, 2011

[6] Instituto Colombiano de Normas Técnicas ICONTEC, Norma Técnica colombiana ISO 50001: 2011, planificación energética, línea base energética, p.8, 2011

[7] Instituto Colombiano de Normas Técnicas ICONTEC, Norma Técnica Colombiana ISO50001:2011, implementación y operación, competencia, formación y toma de conciencia p.9, 2011

[8] Instituto Colombiano de Normas Técnicas ICONTEC, Norma Técnica Internacional ISO 50001: 2011, Implementación y operación, adquisición de servicios de energía, productos, equipos y energía, p.11, 2011

[9] Instituto Colombiano de Normas Técnicas ICONTEC, Norma técnica Internacional ISO 50001:2011, Verificación, evaluación de requisitos legales y de otros requisitos p.12, 2011

[10] Humberto Canto Delgado. "Desarrollo de una cultura de calidad,". Mc Graw Hill interamericana editores S.A México 1997

[11] Norma Técnica colombiana NTC-ISO 9001 editada por el Instituto Colombiano de normas técnicas y certificación Icontec, 2015.

[12] J. M. gallego, L. H Gutiérrez. Sistema nacional de Calidad en Colombia, Banco Interamericano de desarrollo BID, Bogotá 2016

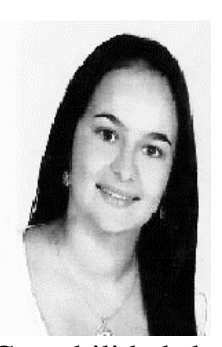

Ángela María Buriticá Macías, Nació en Pereira Risaralda en junio de 1984, bachiller académico fundación gimnasio Pereira, Ingeniera Industrial, Magister en Administración del desarrollo humano y organizacional de la Universidad Tecnológica de Pereira, Docente Facultad de Ciencias Empresariales en el área de Administración y Contabilidad de la Universidad Tecnológica de Pereira desde 2010. Participante en grupos de Investigación en calidad y administración en Facultad de Ciencias empresariales Universidad Tecnológica de Pereira. Participante en proyectos de investigación en competencias laborales, caracterización de compras y proyectos de marketing empresarial, publicación en revista scientia et technica artículo sobre ISO 9001 versión 2015 guía de implementación. Empresaria sector comercio desde año 2011 con especialidad en marketing digital y ventas a través de medios virtuales. Consultora en sistemas de Gestión de calidad, recursos humanos Y marketing digital desde el año 2004.

ORCID: http://orcid.org/0000-0003-0417-6153

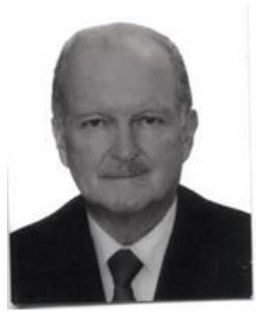

José Germán López Quintero, Ingeniero Electricista, Universidad Tecnológica de Pereira, 2 de marzo de1973

Profesor Titular de Ingeniería Eléctrica de la Universidad Tecnológica de Pereira Vicerrector Académico, 2000 - 2010, de la Universidad Tecnológica de Pereira Vicerrector de Investigaciones, Innovación y Extensión, 2010 - 2014, de la Universidad Tecnológica de Pereira. Rector (encargado) de la Universidad Tecnológica de Pereira .Director Ingeniería Eléctrica desde el año 2015 a la fecha, de la Universidad Tecnológica de Pereira .Par académico Nacional evaluador en la acreditación institucional de alta calidad de universidades y en programas de ingeniería en Colombia, miembro de juntas directivas y Consejo directivos de organizaciones de innovación y desarrollo tecnológico como CIDET, COCIER, WEC Colombia, CIGRÉ Colombia, y de organizaciones gremiales como ACIEM,

Ha publicado varios libros y artículos en revistas especializadas nacionales

ORCID: http://orcid.org/0000-0001-5931-1397

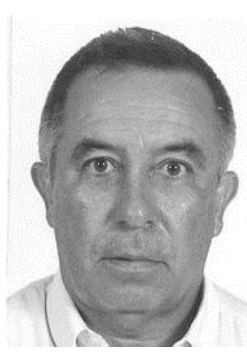

Carlos Alberto Buriticá Noreña, Nació en la ciudad de Manizales, Caldas ,Colombia en el año de 1952 ,bachiller en el Instituto Universitario de Caldas en el año de 1970, Tecnólogo Electricista de la Universidad Tecnológica de Pereira en 1974, Laboro durante 3 años en la Siderúrgica Paz de Rio en Belencito, Boyacá , Colombia como coordinador de mantenimiento realizando la programación Pert y Gantt para la empresa, Ingeniero electricista de la Universidad Tecnológica de Pereira en el año 1979, asistente de proyectos multinacional Papeles Nacionales, 1981 _ 2000 laboro para la empresa textil Coats Cadena en la ciudad de Pereira como jefe de ingeniería, durante 5 años, director de producción 10 años realizando la modernización de la planta, Gerente de operaciones para el área Andina en la misma empresa desde la ciudad de Valencia, Carabobo, Venezuela, desde el 2000 docente de planta en la facultad de Ciencias Empresariales de la Universidad Tecnológica de Pereira, en las áreas de Producción y Aseguramiento de la calidad, fundador del organismo de certificación de sistemas de calidad de la universidad tecnológica, asesor en sistemas de gestión de calidad, medio ambiente y seguridad y salud en el trabajo.

ORCID: https://orcid.org/0000-0002-8910-3000 